\title{
Assessing the Nature of Chiral-Induced Spin Selectivity by Magnetic Resonance
}

\author{
A. Chiesa, M. Chizzini, E. Garlatti, E. Salvadori, F. Tacchino, P. Santini, I. Tavernelli, R. Bittl, M. Chiesa, \\ R. Sessoli, and S. Carretta*
}

Cite This: J. Phys. Chem. Lett. 2021, 12, 6341-6347

Read Online

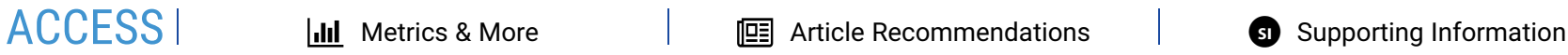

ABSTRACT: Understanding chiral-induced spin selectivity (CISS), resulting from charge transport through helical systems, has recently inspired many experimental and theoretical efforts but is still the object of intense debate. In order to assess the nature of CISS, we propose to focus on electron-transfer processes occurring at the single-molecule level. We design simple magnetic resonance experiments, exploiting a qubit as a highly sensitive and coherent magnetic sensor, to provide clear signatures of the acceptor polarization. Moreover, we show that information could even be obtained from time-resolved electron paramagnetic resonance experiments on a randomly oriented solution of molecules. The proposed experiments will unveil the role of chiral linkers in electron transfer and could also be exploited for quantum computing applications.

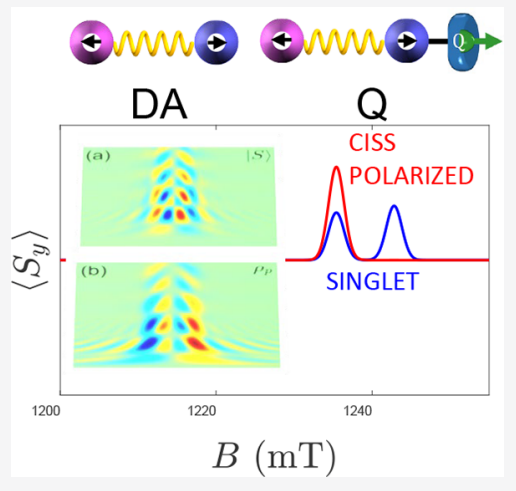

$\mathrm{C}$ harge displacement through chiral systems has been suggested as a resource for spintronics devices and as the driving force of many biological reactions. ${ }^{1-3}$ This has led to huge research efforts, mainly focused on the detection of a spinpolarized current ${ }^{4}$ filtered by chiral molecules, a phenomenon known as chiral-induced spin selectivity (CISS). Transport experiments were done on self-assembled monolayers of chiral $(\chi)$ molecules or on individual molecules addressed by atomic force microscopy. ${ }^{5-8}$ Additional studies revealed polarization also in very different contexts, in which no steady-state current flows through chiral molecules (see the Supporting Information and refs 2 and 9-14). In parallel, various theoretical models have been put forward, ${ }^{15-31}$ but a comprehensive, even qualitative, description of this widespread phenomenon is lacking. ${ }^{8}$

To shed light on the origin of CISS and build a satisfactory theoretical model, we still miss some detailed information on the spin wave function, after an electron has crossed a chiral bridge. This can be achieved by simplifying the experimental setup and focusing on qualitative features, emerging directly from chiral molecules. In particular, electron-transfer (ET) processes through a chiral bridge linking a donor and an acceptor (D- $\chi$ A in the following, see bottom inset of Figure 1) may serve as the ideal platform to understand this phenomenon, in which all other complex elements (such as leads, interfaces, and substrates) have been removed. Recently, a minimal model of ET in chiral environments was proposed, in which the bridge was included via an effective spin-orbit interaction. This leads to no local spin polarization on $\mathrm{D} / \mathrm{A}$ starting from the singlet state precursor obtained by photoexcitation $(\mathrm{PE}) .^{32}$ In contrast,

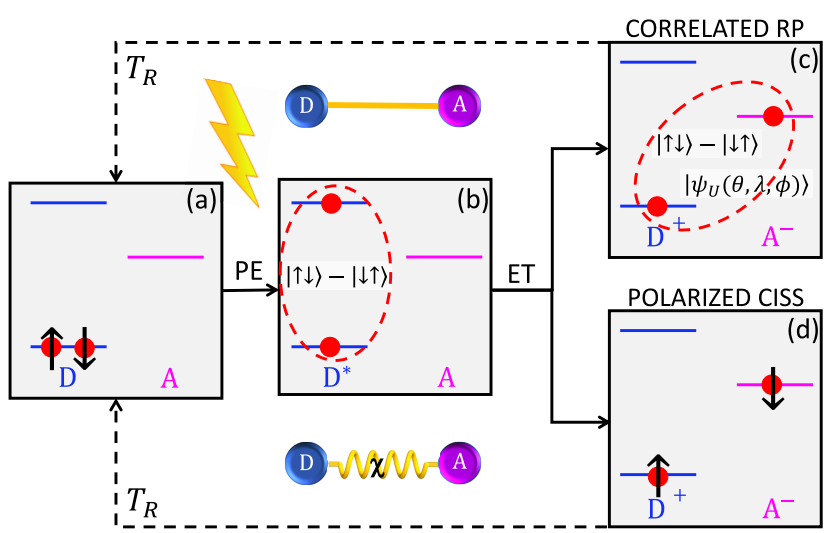

Figure 1. Scheme of the electron-transfer mechanism: (a) singlet initial state on the donor D (with two electrons both in the ground orbital). Photoexcitation (PE) brings it to the $\mathrm{D}^{*} \mathrm{~A}$ singlet state in which one electron is excited (b), but the pair is still in an entangled state (dashed circle). After electron transfer (ET) of the excited electron to the acceptor (A), the final state is still either a correlated radical pair (RP, c) or a polarized state after transfer through a chiral bridge (d). Recombination to the initial singlet (or to the triplet) state occurs on a time scale $T_{\mathrm{R}}$ (dashed arrows). Top (bottom) inset: Scheme of the DA radical pair, linked by a linear (chiral) bridge.

Received: May 5, 2021

Accepted: June 21, 2021

Published: July 6, 2021

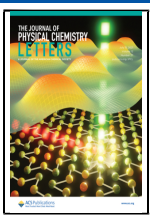


(a)
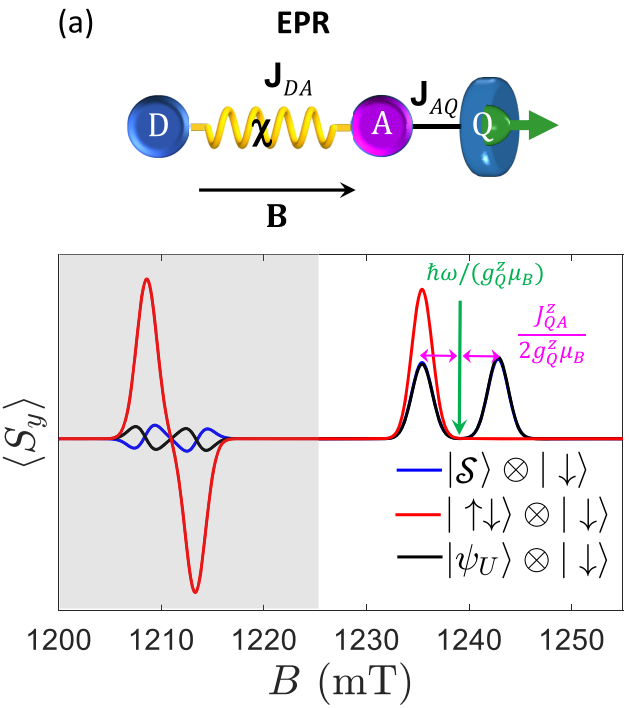

(b)

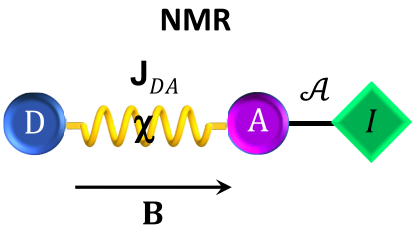

B

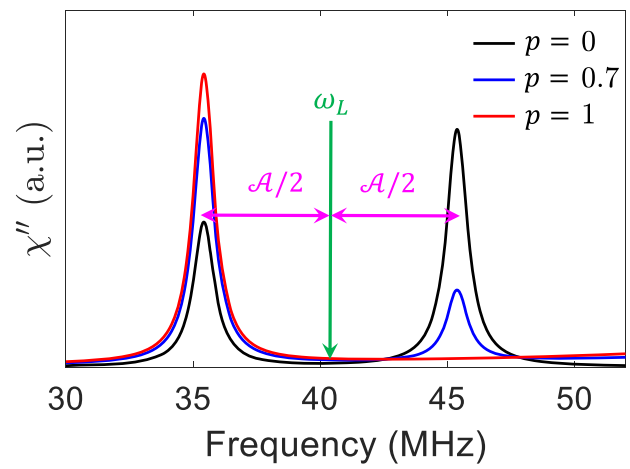

Figure 2. (a) Q-band TR-EPR spectrum of a $\mathrm{D}^{+}-\chi-\mathrm{A}^{-}-\mathrm{Q}$ system (sketched on top) with the chiral axis aligned parallel to the external field (integrated from 100 to $300 \mathrm{~ns}$ ) and for different initial states of the radical pair (with the qubit always in $|\downarrow\rangle$ ): singlet (blue line), corresponding to transfer along a linear bridge without CISS effect; fully polarized state (on both donor and acceptor, red); unpolarized state $\left|\psi_{\mathrm{U}}\right\rangle$ as suggested in ref 32 , black. The grayshaded area represents the signal from the donor-acceptor, while at larger field the absorption peaks are due to the qubit. (b) NMR spectrum as a function of frequency, probing nuclear excitations on a nuclear spin $1 / 2$ (e.g., a ${ }^{19} \mathrm{~F}$, Larmor frequency $\nu_{\mathrm{L}} \approx 40 \mathrm{MHz}$ at $1 \mathrm{~T}$ ) coupled by hyperfine interaction to the donor. The different intensity of the two peaks for $p=0$ is due to different matrix elements for the two transitions. Variance from the $p$ $=0$ behavior directly measures the acceptor polarization. Parameters: $h \nu=34 \mathrm{GHz}, J_{\mathrm{AQ}}^{z} \approx 200 \mathrm{MHz}, r_{\mathrm{DA}}=25 \AA, r_{\mathrm{AQ}}=8 \AA, \mathcal{A}=10 \mathrm{MHz}, g_{1,2}=g_{\mathrm{e}} \mp \Delta g /$ 2 , with $\Delta g=0.002, \mathbf{g}_{Q}=(1.98,1.98,1.96)$, as typical for $\mathrm{VO}^{2+}$ or $\mathrm{Ti}^{3+},{ }^{42} T_{1}=2 \mu \mathrm{s}, T_{2}=0.5 \mu \mathrm{s}$, and $T_{\mathrm{R}}=10 \mu \mathrm{s}$. Inhomogeneous broadening of the parameters is included by a Gaussian broadening of the peaks with fwhm $2.35 \mathrm{mT}$. To generalize our analysis, we did not include parameters of a specific qubit, such as hyperfine interaction.

experiments on photosystem-I ${ }^{1,33}$ have demonstrated a spin polarization occurring also in ET.

We propose here simple experiments to unambiguously distinguish the two situations, thus finally elucidating the nature of CISS, by answering the question: is the electron spin polarized after ET through the chiral bridge? The experiments are based on using a highly coherent qubit $(\mathrm{Q})$, coupled to the acceptor in a D- $\chi$-A-Q setup, as a local probe of this polarization transfer and time-resolved electron paramagnetic resonance (TR-EPR) as the experimental tool. By acting as an external and local sensor, the qubit gives direct access to the acceptor polarization, without influencing the ET process. This provides a unique means to assess the nature of CISS at the single-molecule level, much more directly compared to previous setups, ${ }^{1}$ where many additional ingredients could somehow obscure the role of the $\chi$ unit. The second experiment we propose probes the qubit state after polarization has been coherently transferred from A to Q by an appropriate pulse sequence. We show that both approaches yield unambiguous fingerprints of the polarization of the acceptor if implemented on an oriented solution of $\mathrm{D}-\chi-\mathrm{A}-\mathrm{Q}$ molecules. Moreover, we demonstrate by numerical simulations that features of CISS can also be observed in TR-EPR spectra of a much simpler experimental setup consisting of a randomly oriented ensemble of D- $\chi$-A molecules. The know-how reached by performing the proposed experiments will be the starting point to develop a sound theoretical model of CISS.

$D-\chi$-A System. We consider the following experimental scenario, schematically shown in Figure 1: the donor, initially in a doubly occupied ground state (panel a) is photoexcited to the $\mathrm{D}^{*}-\chi$-A singlet state (b). Then, ET yields the chargeseparated state $\mathrm{D}^{+}-\chi-\mathrm{A}^{-}$. In the case of a linear bridge linking donor and acceptor (top inset), this is still a two-electron singlet state (Figure 1c). Our aim is to compare this situation, typical of a spin-correlated radical pair (RP) ${ }^{34-40}$ with that in the presence of a chiral bridge. In particular, in the experiments proposed below we consider the following charge-separated states:

(1) A singlet state, $|\mathcal{S}\rangle=(|\uparrow \downarrow\rangle-|\downarrow \uparrow\rangle) / \sqrt{2}$, typical of ET through a linear achiral bridge.

(2) A polarized state, represented by the density matrix $\rho_{p}=$ $\frac{1+p}{2}|\uparrow \downarrow\rangle\left\langle\uparrow \downarrow\left|+\frac{1-p}{2}\right| \downarrow \uparrow\right\rangle\langle\downarrow \uparrow|$ (with $-1 \leq p \leq 1$ and $\neq 0)$. Here $p=-2 \operatorname{Tr}\left[\rho_{p} S_{z A}\right]$ is the final polarization of the acceptor. This state (represented in Figure 1d for $p=1$ ) could result from spin selective ET through a chiral bridge, as found in measurements on photosystem- $\mathrm{I}^{33}$ and in other experiments, $^{9-11,13,41}$ where charge polarization was induced by application of an electric field, thus making these situations somewhat similar to ET.

(3) A non-Boltzmann but nonpolarized (correlated) state $\left|\psi_{U}\right\rangle$ (Figure 1c) resulting from a coherent rotation of the transferred electron belonging to $|\mathcal{S}\rangle$, as proposed in ref 32 . The most general form of this state is given by $\left|\psi_{U}\right\rangle=$ $\cos \frac{\theta}{2} \frac{\mathrm{e}^{\mathrm{i}(\lambda+\phi)}|\uparrow \downarrow\rangle-|\downarrow \uparrow\rangle}{\sqrt{2}}-\sin \frac{\theta}{2} \frac{\mathrm{e}^{\mathrm{i} \lambda}|\uparrow \uparrow\rangle+\mathrm{e}^{\mathrm{i} \phi}|\downarrow \downarrow\rangle}{\sqrt{2}}$. One can easily check that $\left|\psi_{U}\right\rangle$ does not give any local (one-)polarization, i.e., $\left\langle\psi_{U}\right| S_{z D}$ $\left|\psi_{U}\right\rangle=\left\langle\psi_{U}\left|S_{z A}\right| \psi_{U}\right\rangle=0$.

The three possible ET outputs are clearly discriminated by the experiments proposed below.

Detecting Polarization Using a Qubit Sensor. To detect spin imbalance in the $\mathrm{D}^{+}-\chi-\mathrm{A}^{-}$unit, we propose the use of a qubit, i.e., a paramagnetic $S=1 / 2$ center showing long coherence. Molecular spin qubits are very promising sensors ${ }^{43}$ thanks to the very long coherence times they can reach if properly chemically engineered. ${ }^{44-58}$ In addition, the capability to link these qubits to other units make them ideal candidates for the proposed architecture. As a model example, we consider a qubit 
based on the $\mathrm{VO}^{2+}$ unit, which has already demonstrated remarkable coherence even at room temperature. ${ }^{50}$ Interestingly, a setup consisting of a chain of three radicals was studied in refs 59-64.

After ET, the whole D- $\chi$-A-Q system is described by the following spin Hamiltonian:

$$
H_{\mathrm{S}}=\mu_{B} \sum_{i=\mathrm{D}, \mathrm{A}, \mathrm{Q}} \mathbf{s}_{i} \cdot \mathbf{g}_{i} \cdot \mathbf{B}+\sum_{i, j} \mathbf{s}_{i} \cdot \mathbf{J}_{i j} \cdot \mathbf{S}_{j}
$$

where the first term models the interaction of each of the three spins with an external magnetic field, while the second describes the magnetic dipole-dipole interaction between $\mathrm{D}-\mathrm{A}$ and $\mathrm{A}-$ $\mathrm{Q}$. We assume the point-dipole approximation, leading to $\mathbf{J}_{i j}=\left[\mathbf{g}_{i}\right.$ $\left.\cdot \mathbf{g}_{j}-3\left(\mathbf{g}_{i} \cdot \mathbf{r}_{i j}\right)\left(\mathbf{g}_{j} \cdot \mathbf{r}_{i j}\right)\right] \mu_{B}{ }^{2} / r_{i j}{ }^{3}$, and in the following we consider for simplicity a linear 3-spin chain (see Figure 2a), with $r_{D A}=25$ $\AA$ and isotropic $g_{\mathrm{D}, \mathrm{A}}$. As demonstrated by simulations reported in the Supporting Information, possible additional isotropic exchange contributions to $\mathrm{J}_{\mathrm{AQ}}$ do not alter our conclusions, provided that $J_{\mathrm{AQ}}^{x, y}$ is sufficiently smaller than $\left|g_{\mathrm{A}}-g_{\mathrm{Q}}^{z}\right| \mu_{B} B$ to make the initial state of the qubit factorized from that of the acceptor. We note, in turn, that a stronger $\mathrm{A}-\mathrm{Q}$ coupling could be compensated either by increasing $B$ or by choosing a different qubit, based for instance on $\mathrm{Cu}^{2+}, 52$ yielding a larger $\left|g_{\mathrm{A}}-g_{\mathrm{Q}}^{z}\right|$. In this regime, $\mathrm{Q}$ acts as a coherent quantum sensor which does not perturb the RP but only detects local spin polarization. At the same time, $J_{\mathrm{AQ}}^{z}$ should be larger than the line width corresponding to $\mathrm{Q}$ excitations. For instance, by choosing $\mathrm{fwhm}=2.35 \mathrm{mT}$ (a conservative estimate for typical transition metal ion based qubits ${ }^{42,49}$ ), these conditions are easily fulfilled with $6 \AA \lesssim r_{A Q} \lesssim 11 \AA$ and working in Q-band. We thus fix $r_{\mathrm{AQ}}=$ $8 \AA$ in the following simulations.

Two different experiments exploiting the qubit as a sensor of the acceptor polarization are proposed. The first consists of TREPR measurements recorded immediately after ET. To simulate TR-EPR spectra, we compute the time evolution of the system density matrix by integrating the Liouville equation $\dot{\rho}=$ $-\mathrm{i}\left[\frac{1}{\hbar} \tilde{H}+\mathrm{i} \tilde{R}+\mathrm{i} \tilde{K}\right] \rho$, where $\rho$ is the system density matrix in the rotating frame and $\tilde{H}, \tilde{R}$, and $\tilde{K}$ are superoperators associated with the system Hamiltonian (including also a continuous-wave oscillating field) and with phenomenological incoherent mechanisms. These are (i) relaxation, (ii) dephasing, and (iii) recombination (see the Supporting Information and, for example, refs 36-40 and 65), parametrized by the characteristic times $T_{1}, T_{2}$, and $T_{R}$, respectively, treated as independent for each of the three spins. ${ }^{61}$ We use conservative values (even at room temperature) for each of the three spins of $T_{1} \approx 2 \mu \mathrm{s}, T_{2} \approx$ $0.5 \mu \mathrm{s}$, and $T_{\mathrm{R}} \approx 10 \mu \mathrm{s}$. $^{37,38,50}$ The precise value of these parameters yields only a broadening of the peaks in the fielddependent spectrum and a damping in the time evolution, as shown by detailed simulations in the Supporting Information. However, as far as these characteristic times are above a few hundred nanoseconds, they practically do not affect our conclusions. The recorded signal then corresponds to $\left\langle S_{y}(t\right.$, $B)\rangle=\operatorname{Tr}\left[\sum_{i} S_{y i} \rho(t)\right]$. In order to unambiguously unveil the nature of CISS, we consider oriented D- $\chi$-A-Q molecules, with the static field parallel to the chiral axis, $z$ (experimentally achieved for instance by poling, thanks to the large electric dipole moment typical of chiral molecules based on oligopeptides $^{24,66-68}$ ).

The three states $(1-3)$ give distinct TR-EPR signals, as shown in the time-integrated spectra of Figure 2a. In particular, we note that different (unpolarized) states, such as $|\mathcal{S}\rangle$ or $\left|\psi_{U}(\theta, \phi, \lambda)\right\rangle$, modify the radical-pair spectrum (black vs blue curves in the gray-shaded area) but not the qubit response (right part of the spectrum), which is affected only by $\left\langle S_{z A}\right\rangle$. The qubit absorption peak close to $\sim 1.24 \mathrm{~T}$ is split by the interaction with the acceptor. If the latter is completely polarized (e.g., in $|\downarrow\rangle_{\mathrm{A}}$ state, $p$ $=1$ ), a single peak appears, corresponding to the $|\uparrow \downarrow \downarrow\rangle \rightarrow|\uparrow \downarrow \uparrow\rangle$ transition. ${ }^{69}$ Conversely, an unpolarized DA state induces the additional excitation corresponding to $|\uparrow\rangle_{A}$ with approximately the same intensity (apart from slightly different matrix elements or thermal population). Note that in the present simulation, $\left\langle S_{y}(t, B)\right\rangle$ shows a weak time dependence (see the Supporting Information), thus making the choice of the time window of integration not crucial.

Similar information can be obtained by broadband NMR spectroscopy, as reported in Figure $2 \mathrm{~b}$. We consider, in this case, a nuclear spin $I=1 / 2$ (such as ${ }^{19} \mathrm{~F}$ ) coupled to A by (isotropic) hyperfine interaction $\mathcal{A} \mathbf{S}_{A} \cdot \mathbf{I}$. The NMR absorption signal $\left(\chi^{\prime \prime}\right)$ is shown as a function of frequency, in the range corresponding to the excitation of nucleus $I$, split by hyperfine interaction with A. Again, the probe is only sensitive to $\left\langle S_{z A}\right\rangle$ and weakly perturbs the system, thus giving direct access to the acceptor polarization. $^{70}$

Partial polarization leads to intermediate situations (blue curve in Figure $2 \mathrm{~b}$ and the Supporting Information), thus making the relative intensity of the two peaks a measure of spin polarization. Remarkably, both for EPR and NMR experiments, this feature is not hampered by performing the experiment at high temperature, which only induces an overall attenuation of the signal (see simulation in the Supporting Information). Moreover, opposite polarization (arising in model 2 by changing the enantiomer) yields inversion of the intensity of the two peaks, thus providing direct proof of the occurrence of CISS, in contrast to other polarization mechanisms such as chemically induced dynamic electron polarization (CIDEP). ${ }^{71}$

The second experiment is based on a sequence of pulses properly designed to coherently transfer polarization from A to $\mathrm{Q}$, followed by EPR measurement of the final state of the system. The sequence is reported in Figure 3a,b: it consists of a first $\pi$ pulse on A (conditioned by $Q$ state, purple arrow), followed by a $\pi$-pulse on $\mathrm{Q}$ conditioned by the state of A (green arrow). Starting with the qubit in a complete mixture, $\rho_{\mathrm{Q}}=\mathbb{1} / 2$ (hightemperature limit), and a fully polarized acceptor state, this sequence completely transfers polarization to $\mathrm{Q}$ leaving $\mathrm{A}$ in an unpolarized mixture. The final state of the system can be measured by EPR after the pulses. The resulting $\left\langle S_{y}\right\rangle$ is reported in Figure $3 c$, for the case of an initially polarized acceptor state (red, blue lines), compared to an initially unpolarized one $\left(\left|\psi_{U}\right\rangle\right.$ or $|\mathcal{S}\rangle$, black). The latter give a very weak signal, both at low field (from the radical pair) and at high field (from the qubit). Conversely, starting from a polarized $\left|\downarrow_{D} \uparrow_{A}\right\rangle$ state, the final state is also polarized on both $\mathrm{D}$ and $\mathrm{Q}$. The peaks corresponding to excitations of $Q$ and $D$ are then split by $J_{A Q}$ and $J_{D A}$, respectively (the latter being much smaller and hence not visible in Figure $3 c)$. Note that by changing the enantiomer the absorption spectrum changes sign, due to reversal of the initial state polarization (blue trace in Figure 3c). This provides clear proof that the net spin polarization arises from CISS. Hence, a qubit (or a nuclear spin) weakly interacting with a D- $\chi$-A unit is the ideal probe of the spin imbalance on the DA pair and would unveil the nature of CISS. We also point out that the proposed platform is robust even at room temperature, a condition in 

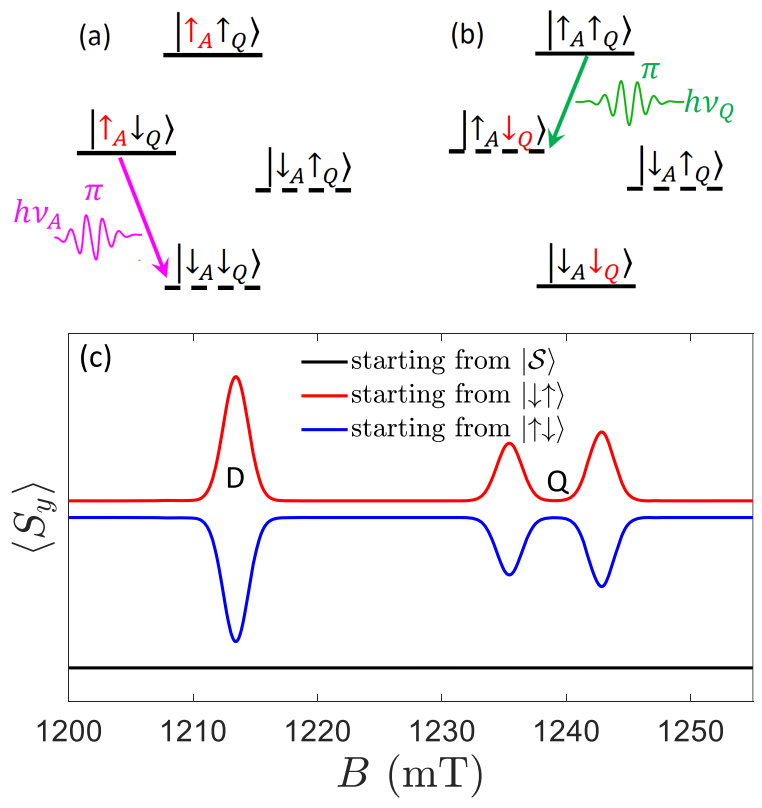

Figure 3. Polarization transfer to the qubit probe: (a) and (b) Pulse sequence implementing the scheme on the AQ pair (D has opposite polarization compared to $A$ and is not affected by the pulses; that is, rotations of $\mathrm{A}$ are independent from the state of $\mathrm{D}$ ). Full (dashed) lines indicate occupied (empty) states, initially with fully polarized A, finally with polarization transferred to $\mathrm{Q}$ (red arrow). The two pulses on $\mathrm{A}$ (Q) are indicated by a purple (green) arrow. (c) TR-EPR spectrum (integrated from 100 to $300 \mathrm{~ns}$ ) after application of the polarization transfer sequence for an unpolarized state (black line) or for a spin polarized one (red or blue, depending on the polarization), as expected after CISS induced by each of the two enantiomers. Transitions involving excitations of $\mathrm{D}(\mathrm{Q})$ are represented by peaks at low (high) field.

which many $\chi$ units keep large polarization efficiency ${ }^{2}$ and $\mathrm{VO}^{2+}$ qubits maintain remarkable coherence. ${ }^{50}$

TR-EPR on $D-\chi-A$ in Solution. In order to facilitate the first experimental attempts, we further simplify our setup and consider TR-EPR experiments ${ }^{36-38,62,63,72}$ on an isotropic solution of $\mathrm{D}-\chi$-A molecules. It was recently pointed out ${ }^{73}$ that angular average on the initial state cancels the most clear signature of CISS. However, we find that, in the presence of an anisotropic dipolar DA interaction, characteristic features of CISS are already present in the spectrum of an isotropic solution of $\mathrm{D}-\chi$-A molecules. In particular, the different initial states discussed in the previous sections lead to different time evolutions and hence to significantly different spectra at short times. As an example, Figure 4 shows simulated TR-EPR spectra at $9.8 \mathrm{GHz}$ (X-band), along with cuts for specific time/field windows. We immediately note that at short times the $|\mathcal{S}\rangle$ state (panel a) gives an opposite pattern of maxima and minima, compared with $\rho_{p}$ (panel b). This also emerges from the shorttime spectra, as a function of $B$ (panel c), and from the time dependence, reported in panel $\mathrm{d}$ for $B$ corresponding to the first pronounced peak. The different order of maxima and minima as a function of $B$ can be understood by considering the form of the initial state along different directions. An illustrative diagram of the (practically factorized) eigenstates is shown in the inset of Figure $4 \mathrm{~d}$, with levels labeled in order of increasing energy from 1 to 4 and allowed transitions indicated by dashed lines. Note that the corresponding gaps and resonance fields are made different by $\mathbf{J}_{\mathrm{DA}}$. An initial $|\mathcal{S}\rangle$ state shows spherical symmetry
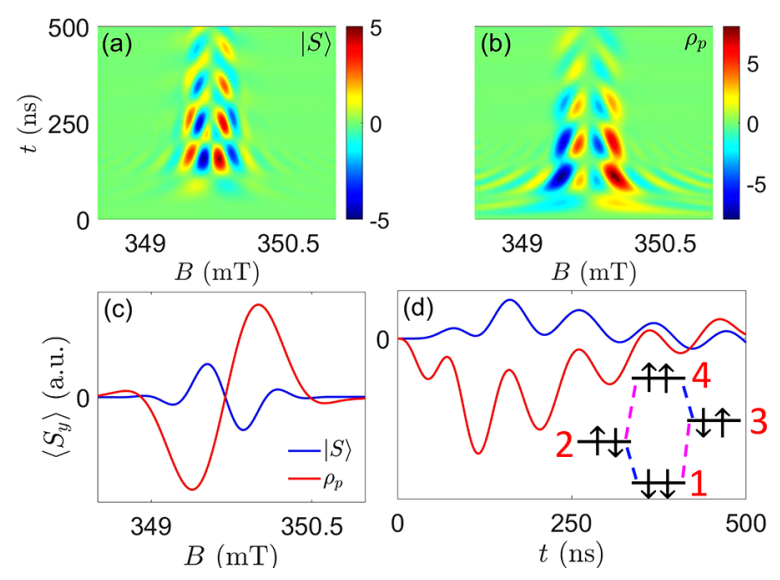

Figure 4. TR-EPR on a randomly oriented ensemble of D- $\chi$-A molecules. Parameters: $\Delta g=0.002, r_{\mathrm{DA}}=25 \AA, h \nu=9.8 \mathrm{GHz}$. (a) and (b) Two-dimensional maps of $\left\langle S_{y}(t, B)\right\rangle$ for an initial singlet or polarized state, respectively. (c) Field dependence of the absorption TR-EPR spectrum, integrated in the time-window corresponding to the first maxima-minima in the maps of panels (a) and (b), for the states $|\mathcal{S}\rangle$ , $\rho_{p}$ (with either $p=-1$ or $p=1$, leading to the same result in solution). (d) Time dependence around $B \approx 349.5 \mathrm{mT}$, highlighting the opposite behavior at short times for polarized and unpolarized states. Simulations include relaxation, dephasing, and recombination of the radical pair, with $T_{1}=2 \mu \mathrm{s}, T_{2}=0.5 \mu \mathrm{s}, T_{R}=10 \mu \mathrm{s}$ (in the singlet-triplet $\mathrm{RP}$ basis, see the Supporting Information), and Gaussian broadening with $\mathrm{fwhm}=0.15 \mathrm{mT} .^{36}$ Inset: schematic energy-level diagram, with states practically corresponding to eigenstates of $S_{z i}$. Allowed EPR transitions of D (A) are indicated by blue (purple) dashed lines.

and hence keeps the same form in any direction. The static Hamiltonian induces partial population transfer from the dark $|\mathcal{S}\rangle$ state to the symmetric superposition $\left|T_{0}\right\rangle=(|\uparrow \downarrow\rangle+\mid \downarrow$ $\uparrow\rangle) / \sqrt{ } 2$. Hence, the EPR signal shows emission lines for $2-1$ and 3-1 transitions and absorptions for 2-4 and 3-4. Conversely, an initial $\rho_{p}$ state (polarized along the chiral axis) is strongly anisotropic. For a generic orientation of the molecule with respect to the external field (which defines the quantization axis) such a state has sizable components on states $|\uparrow \uparrow\rangle$ and $|\downarrow \downarrow\rangle$. If these components are larger than that on $\left|T_{0}\right\rangle$, we get emission lines for 4-2 and 4-3 transitions and absorption for 1-2 and $1-3$. This situation (opposite to that of the singlet) dominates on the spherical average (see the Supporting Information for details). Then, the order of maxima and minima is determined by the sign of the spin-spin interaction (fixed by considering a dipolar coupling).

We finally note that, because the spectrum in panel $b$ is the same for any choice of $p$, this measurement does not probe the acceptor polarization but distinguishes a factorized state $\rho_{p}$ from a singlet. As shown in the Supporting Information, unpolarized states $\left|\psi_{U}\right\rangle$ give different patterns, intermediate between $|\mathcal{S}\rangle$ and $\rho_{p}$, thus requiring, in general, a preliminary characterization of the system Hamiltonian to clearly reveal CISS.

Identification of a Suitable $D-\chi-A-Q$. The identification of a suitable $\mathrm{D}-\chi-\mathrm{A}-\mathrm{Q}$ requires the optimization of many factors, including ET efficiency and stability of the helicoidal structure in solution, but appears within reach. Considering the individual building blocks, D-A dyads providing long-lived radical pairs are available in the literature. ${ }^{74,75}$ The most commonly used acceptors are $\mathrm{C}_{60}$ or derivatives of naphthalenediimide, whereas pyrene, oligophenylene-vinylene, and tetrathiafulvalene are suitable donors. Linkers employed in these dyads are usually short and strongly conjugated and thus significantly more 
conductive than foldamers based on polypeptides commonly used to detect CISS in transport measurements. ${ }^{1,2}$ Interestingly, the strong dipole moment of $\alpha$ helices has been shown to enhance intramolecular ET. Long distance ET, up to $5 \mathrm{~nm}$, has been instead observed in D- $\chi$-A units based on helically folded oligoamide of 8 -amino-2-quinolinecarboxylic acid. ${ }^{76}$ However, both forms of handedness are present and interconversion is relatively fast in solution. Nevertheless, racemization can be hampered in more rigid helicoidal scaffolds such as helicene. Condensation of a D-A unit with a radical as a qubit has been recently achieved. ${ }^{64,77}$ In our case, where pulses separately addressing the photogenerated radical and the permanent qubit are required, transition metal-based qubits with long coherence ${ }^{42,44-58}$ are preferred because of their $g$ value differing from 2 , as discussed above.

In conclusion, we have proposed simple magnetic resonance experiments exploiting a qubit as a probe of the acceptor polarization in electron-transfer processes through a chiral bridge. These experiments will ultimately unravel the nature of chiral-induced spin selectivity at the single-molecule level. We finally note that, by applying the proposed sequence for polarization transfer, the CISS effect could be exploited for initialization and read-out of the qubit state, an alternative to optical initialization recently achieved in a $\mathrm{Cr}^{4+} S=1$ complex. $^{78}$ This is a crucial step toward the physical implementation of quantum computers. A CISS-based approach would be more costly in terms of chemical engineering, but it could reveal tremendous potential. Indeed, the photoexcitation step could be replaced by electrically induced CISS-ET and combined with electric read-out.

\section{ASSOCIATED CONTENT}

\section{SI Supporting Information}

The Supporting Information is available free of charge at https://pubs.acs.org/doi/10.1021/acs.jpclett.1c01447.

Overview of state-of-the-art experiments on CISS, details on the simulation of time-resolved EPR spectra on D- $\chi$-A$\mathrm{Q}$ and $\mathrm{D}-\chi-\mathrm{A}$ systems, additional simulations with different parameter set and including the effect of isotropic exchange and of experimental imperfections, and details on the calculation of NMR spectra (PDF)

\section{AUTHOR INFORMATION}

\section{Corresponding Author}

S. Carretta - Dipartimento di Scienze Matematiche, Fisiche e Informatiche, Università di Parma, I-43124 Parma, Italy; UdR Parma, INSTM, I-43124 Parma, Italy; $\odot$ orcid.org/ 0000-0002-2536-1326; Email: stefano.carretta@unipr.it

\section{Authors}

A. Chiesa - Dipartimento di Scienze Matematiche, Fisiche e Informatiche, Università di Parma, I-43124 Parma, Italy; UdR Parma, INSTM, I-43124 Parma, Italy; (1) orcid.org/ 0000-0003-2955-3998

M. Chizzini - Dipartimento di Scienze Matematiche, Fisiche e Informatiche, Università di Parma, I-43124 Parma, Italy

E. Garlatti - Dipartimento di Scienze Matematiche, Fisiche e Informatiche, Università di Parma, I-43124 Parma, Italy; UdR Parma, INSTM, I-43124 Parma, Italy; 잉oㅇ.org/ 0000-0002-0370-0534
E. Salvadori - Dipartimento di Chimica \& NIS Centre, Università di Torino, I-10125 Torino, Italy; (1) orcid.org/ 0000-0003-4394-9438

F. Tacchino - IBM Quantum, IBM Research-Zurich, 8803 Rüschlikon, Switzerland

P. Santini - Dipartimento di Scienze Matematiche, Fisiche e Informatiche, Università di Parma, I-43124 Parma, Italy; UdR Parma, INSTM, I-43124 Parma, Italy

I. Tavernelli - IBM Quantum, IBM Research-Zurich, 8803 Rüschlikon, Switzerland; ○ orcid.org/0000-0001-56901981

R. Bittl - Freie Universität Berlin, Fachbereich Physik, Berlin Joint EPR Lab, D-14195 Berlin, Germany

M. Chiesa - Dipartimento di Chimica \& NIS Centre, Università di Torino, I-10125 Torino, Italy; 이이.org/ 0000-0001-8128-8031

R. Sessoli - Dipartimento di Chimica "Ugo Schiff" \& INSTM, Università Degli Studi di Firenze, I-50019 Sesto Fiorentino, Italy; () orcid.org/0000-0003-3783-2700

Complete contact information is available at:

https://pubs.acs.org/10.1021/acs.jpclett.1c01447

\section{Notes}

The authors declare no competing financial interest.

\section{ACKNOWLEDGMENTS}

This work received financial support from the Italian Ministry of Education and Research (MUR) through PRIN project 2017 QchiSS "Quantum detection of chiral-induced spin-selectivity at the molecular level" and from the European Union's Horizon 2020 program under Grant Agreement No. 862893 (FETOPEN project FATMOLS). We thank Andrea Cornia, Ron Naaman, Francesco Pineider and Claudia Tomasini for stimulating discussions.

\section{REFERENCES}

(1) Naaman, R.; Paltiel, Y.; Waldeck, D. H. Chiral molecules and the electron spin. Nat. Rev. Chem. 2019, 3, 250-260.

(2) Naaman, R.; Paltiel, Y.; Waldeck, D. H. Chiral Induced Spin Selectivity Gives a New Twist on Spin-Control in Chemistry. Acc. Chem. Res. 2020, 53, 2659-2667.

(3) Metzger, T. S.; Mishra, S.; Bloom, B. P.; Goren, N.; Neubauer, A.; Shmul, G.; Wei, J.; Yochelis, S.; Tassinari, F.; Fontanesi, C.; Waldeck, D. H.; Paltiel, Y.; Naaman, R. The Electron Spin as a Chiral Reagent. Angew. Chem., Int. Ed. 2020, 59, 1653-1658.

(4) Naaman, R; Waldeck, D. H. Spintronics and Chirality: Spin Selectivity in Electron Transport Through Chiral Molecules. Annu. Rev. Phys. Chem. 2015, 66, 263-281.

(5) Nogues, C.; Cohen, S. R.; Daube, S. S.; Naaman, R. Electrical properties of short DNA oligomers characterized by conducting atomic force microscopy. Phys. Chem. Chem. Phys. 2004, 6, 4459-4466.

(6) Xie, Z.; Markus, T. Z.; Cohen, S. R.; Vager, Z.; Gutierrez, R.; Naaman, R. Spin Specific Electron Conduction through DNA Oligomers. Nano Lett. 2011, 11, 4652-4655.

(7) Aragonès, A. C.; Medina, E.; Ferrer-Huerta, M.; Gimeno, N.; Teixidó, M.; Palma, J. L.; Tao, N.; Ugalde, J. M.; Giralt, E.; Díez-Pérez, I.; Mujica, V. Measuring the Spin-Polarization Power of a Single Chiral Molecule. Small 2017, 13, 1602519.

(8) Waldeck, D. H.; Naaman, R.; Paltiel, Y. The spin selectivity effect in chiral materials. APL Mater. 2021, 9, 040902.

(9) Ben Dor, O.; Yochelis, S.; Radko, A.; Vankayala, K.; Capua, E.; Capua, A.; Yang, S.-H.; Baczewski, L. T.; Parkin, S. S. P.; Naaman, R.; Paltiel, Y. Magnetization switching in ferromagnets by adsorbed chiral molecules without current or external magnetic field. Nat. Commun. $2017,8,14567$. 
(10) Banerjee-Ghosh, K.; Ben Dor, O.; Tassinari, F.; Capua, E.; Yochelis, S.; Capua, A.; Yang, S.-H.; Parkin, S. S. P.; Sarkar, S.; Kronik, L.; Baczewski, L. T.; Naaman, R.; Paltiel, Y. Separation of enantiomers by their enantiospecific interaction with achiral magnetic substrates. Science 2018, 360, 1331-1334.

(11) Kumar, A.; Capua, E.; Kesharwani, M. K.; Martin, J. M. L.; Sitbon, E.; Waldeck, D. H.; Naaman, R. Chirality-induced spin polarization places symmetry constraints on biomolecular interactions. Proc. Natl. Acad. Sci. U. S. A. 2017, 114, 2474-2478.

(12) Ziv, A.; Saha, A.; Alpern, H.; Sukenik, N.; Baczewski, L. T.; Yochelis, S.; Reches, M.; Paltiel, Y. AFM-Based Spin-Exchange Microscopy Using Chiral Molecules. Adv. Mater. 2019, 31, 1904206.

(13) Smolinsky, E. Z. B.; Neubauer, A.; Kumar, A.; Yochelis, S.; Capua, E.; Carmieli, R.; Paltiel, Y.; Naaman, R.; Michaeli, K. Electric Field-Controlled Magnetization in GaAs/AlGaAs HeterostructuresChiral Organic Molecules Hybrids. J. Phys. Chem. Lett. 2019, 10, 11391145.

(14) Naaman, R.; Paltiel, Y.; Waldeck, D. H. Chiral Molecules and the Spin Selectivity Effect. J. Phys. Chem. Lett. 2020, 11, 3660-3666.

(15) Guo, A.-M.; Sun, Q.-F. Spin-dependent electron transport in protein-like single-helical molecules. Proc. Natl. Acad. Sci. U. S. A. 2014, $111,11658-11662$.

(16) Varela, S.; Mujica, V.; Medina, E. Effective spin-orbit couplings in an analytical tight-binding model of DNA: Spin filtering and chiral spin transport. Phys. Rev. B: Condens. Matter Mater. Phys. 2016, 93, 155436.

(17) Pan, T.-R.; Guo, A.-M.; Sun, Q.-F. Spin-polarized electron transport through helicene molecular junctions. Phys. Rev. B: Condens. Matter Mater. Phys. 2016, 94, 235448.

(18) Matityahu, S.; Utsumi, Y.; Aharony, A.; Entin-Wohlman, O.; Balseiro, C. A. Spin-dependent transport through a chiral molecule in the presence of spin-orbit interaction and nonunitary effects. Phys. Rev. B: Condens. Matter Mater. Phys. 2016, 93, 075407.

(19) Díaz, E.; Domínguez-Adame, F.; Gutierrez, R.; Cuniberti, G.; Mujica, V. Thermal Decoherence and Disorder Effects on ChiralInduced Spin Selectivity. J. Phys. Chem. Lett. 2018, 9, 5753-5758.

(20) Geyer, M.; Gutierrez, R.; Mujica, V.; Cuniberti, G. ChiralityInduced Spin Selectivity in a Coarse-Grained Tight-Binding Model for Helicene. J. Phys. Chem. C 2019, 123, 27230-27241.

(21) Zöllner, M. S.; Varela, S.; Medina, E.; Mujica, V.; Herrmann, C. Insight into the Origin of Chiral-Induced Spin Selectivity from a Symmetry Analysis of Electronic Transmission. J. Chem. Theory Comput. 2020, 16, 2914-2929.

(22) Varela, S.; Zambrano, I.; Berche, B.; Mujica, V.; Medina, E. Spinorbit interaction and spin selectivity for tunneling electron transfer in DNA. Phys. Rev. B: Condens. Matter Mater. Phys. 2020, 101, 241410.

(23) Medina, E.; González-Arraga, L. A.; Finkelstein-Shapiro, D.; Berche, B.; Mujica, V. Continuum model for chiral induced spin selectivity in helical molecules. J. Chem. Phys. 2015, 142, 194308.

(24) Michaeli, K.; Naaman, R. Origin of Spin-Dependent Tunneling Through Chiral Molecules. J. Phys. Chem. C 2019, 123, 17043-17048.

(25) Geyer, M.; Gutierrez, R.; Cuniberti, G. Effective Hamiltonian model for helically constrained quantum systems within adiabatic perturbation theory: Application to the chirality-induced spin selectivity (CISS) effect. J. Chem. Phys. 2020, 152, 214105.

(26) Fransson, J. Chirality-Induced Spin Selectivity: The Role of Electron Correlations. J. Phys. Chem. Lett. 2019, 10, 7126-7132.

(27) Dalum, S.; Hedegård, P. Theory of Chiral Induced Spin Selectivity. Nano Lett. 2019, 19, 5253-5259.

(28) Matxain, J. M.; Ugalde, J. M.; Mujica, V.; Allec, S. I.; Wong, B. M.; Casanova, D. Chirality Induced Spin Selectivity of Photoexcited Electrons in Carbon-Sulfur [n]Helicenes. ChemPhotoChem. 2019, 3, $770-777$.

(29) Zhang, L.; Hao, Y.; Qin, W.; Xie, S.; Qu, F. Chiral-induced spin selectivity: A polaron transport model. Phys. Rev. B: Condens. Matter Mater. Phys. 2020, 102, 214303.

(30) Hu, P.-J.; Wang, S.-X.; Gao, X.-H.; Zhang, Y.-Y.; Fang, T.-F.; Guo, A.-M.; Sun, Q.-F. Spin-dependent electron transport along hairpinlike DNA molecules. Phys. Rev. B: Condens. Matter Mater. Phys. 2020, 102, 195406.
(31) Liu, Y.; Xiao, J.; Koo, J.; Yan, B. Chirality-driven topological electronic structure of DNA-like materials. Nat. Mater. 2021, 20, 638.

(32) Fay, T. P. Chirality-Induced Spin Coherence in Electron Transfer Reactions. J. Phys. Chem. Lett. 2021, 12, 1407-1412.

(33) Carmeli, I.; Kumar, K. S.; Heifler, O.; Carmeli, C.; Naaman, R. Spin Selectivity in Electron Transfer in Photosystem I. Angew. Chem., Int. Ed. 2014, 53, 8953-8958.

(34) Stehlik, D.; Bock, C. H.; Petersen, J. Anisotropic electron spin polarization of correlated spin pairs in photosynthetic reaction centers. J. Phys. Chem. 1989, 93, 1612-1619.

(35) Salikhov, K. M.; Bock, C. H.; Stehlik, D. Time Development of Electron Spin Polarization in Magnetically Coupled, Spin Correlated Radical Pairs. Appl. Magn. Reson. 1990, 1, 195-211.

(36) Bittl, R.; Kothe, G. Transient EPR of radical pairs in photosynthetic reaction centers: prediction of quantum beats. Chem. Phys. Lett. 1991, 177, 547-553.

(37) Kothe, G.; Weber, S.; Bittl, R.; Ohmes, E.; Thurnauer, M. C.; Norris, J. R. Transient EPR of light-induced radical pairs in plant photosystem I: observation of quantum beats. Chem. Phys. Lett. 1991, $186,474-480$.

(38) Kothe, G.; Weber, S.; Ohmes, E.; Thurnauer, M. C.; Norris, J. R. Transient EPR of Light-Induced Spin-Correlated Radical Pairs: Manifestation of Zero Quantum Coherence. J. Phys. Chem. 1994, 98, 2706-2712.

(39) Weber, S.; Ohmes, E.; Thurnauer, M. C.; Norris, J. R.; Kothe, G. Light-generated nuclear quantum beats: a signature of photosynthesis. Proc. Natl. Acad. Sci. U. S. A. 1995, 92, 7789-7793.

(40) Weber, S.; Biskup, T.; Okafuji, A.; Marino, A. R.; Berthold, T.; Link, G.; Hitomi, K.; Getzoff, E. D.; Schleicher, E.; Norris, J. R. Origin of Light-Induced Spin-Correlated Radical Pairs in Cryptochrome. J. Phys. Chem. B 2010, 114, 14745-14754.

(41) Ghosh, S.; Mishra, S.; Avigad, E.; Bloom, B. P.; Baczewski, L. T.; Yochelis, S.; Paltiel, Y.; Naaman, R.; Waldeck, D. H. Effect of Chiral Molecules on the Electron's Spin Wavefunction at Interfaces. J. Phys. Chem. Lett. 2020, 11, 1550-1557.

(42) Camargo, L. C.; Briganti, M.; Santana, F. S.; Stinghen, D.; Ribeiro, R. R.; Nunes, G. G.; Soares, J. F.; Salvadori, E.; Chiesa, M.; Benci, S.; Torre, R.; Sorace, L.; Totti, F.; Sessoli, R. Exploring the Organometallic Route to Molecular Spin Qubits: The [CpTi(cot)] Case. Angew. Chem., Int. Ed. 2021, 60, 2588-2593.

(43) Degen, C. L.; Reinhard, F.; Cappellaro, P. Quantum sensing. Rev. Mod. Phys. 2017, 89, 035002.

(44) Zadrozny, J. M.; Niklas, J.; Poluektov, O. G.; Freedman, D. E. Millisecond Coherence Time in a Tunable Molecular Electronic Spin Qubit. ACS Cent. Sci. 2015, 1, 488.

(45) Yu, C.-J.; Graham, M. J.; Zadrozny, J. M.; Niklas, J.; Krzyaniak, M. D.; Wasielewski, M. R.; Poluektov, O. G.; Freedman, D. E. Long Coherence Times in Nuclear Spin-Free Vanadyl Qubits. J. Am. Chem. Soc. 2016, 138, 14678-14685.

(46) Graham, M. J.; Zadrozny, J. M.; Shiddiq, M.; Anderson, J. S.; Fataftah, M. S.; Hill, S.; Freedman, D. E. Influence of Electronic Spin and Spin-Orbit Coupling on Decoherence in Mononuclear Transition Metal Complexes. J. Am. Chem. Soc. 2014, 136, 7623-7626.

(47) Wojnar, M. K.; Laorenza, D. W.; Schaller, R. D.; Freedman, D. E. Nickel(II) Metal Complexes as Optically Addressable Qubit Candidates. J. Am. Chem. Soc. 2020, 142, 14826-14830.

(48) Fataftah, M.; Zadrozny, J. M.; Coste, S. C.; Graham, M. J.; Rogers, D. M.; Freedman, D. E. Employing Forbidden Transitions as Qubits in a Nuclear Spin-Free Chromium Complex. J. Am. Chem. Soc. 2016, 138, 1344.

(49) Atzori, M.; Morra, E.; Tesi, L.; Albino, A.; Chiesa, M.; Sorace, L.; Sessoli, R. Quantum Coherence Times Enhancement in Vanadium(IV)-based Potential Molecular Qubits: the Key Role of the Vanadyl Moiety. J. Am. Chem. Soc. 2016, 138, 11234-11244.

(50) Atzori, M.; Tesi, L.; Morra, E.; Chiesa, M.; Sorace, L.; Sessoli, R. Room-Temperature Quantum Coherence and Rabi Oscillations in Vanadyl Phthalocyanine: Toward Multifunctional Molecular Spin Qubits. J. Am. Chem. Soc. 2016, 138, 2154-2157. 
(51) Ding, Y.-S.; Deng, Y.-F.; Zheng, Y.-Z. The Rise of Single-Ion Magnets as Spin Qubits. Magnetochemistry 2016, 2, 40.

(52) Bader, K.; Dengler, D.; Lenz, S.; Endeward, B.; Jiang, S.-D.; Neugebauer, P.; van Slageren, J. Room temperature quantum coherence in a potential molecular qubit. Nat. Commun. 2014, 5, 5304.

(53) Shiddiq, M.; Komijani, D.; Duan, Y.; Gaita-Ariño, A.; Coronado, E.; Hill, S. Enhancing coherence in molecular spin qubits via atomic clock transitions. Nature 2016, 531, 348-351.

(54) Atzori, M.; Tesi, L.; Benci, S.; Lunghi, A.; Righini, R.; Taschin, A.; Torre, R.; Sorace, L.; Sessoli, R. Spin Dynamics and Low Energy Vibrations: Insights from Vanadyl- Based Potential Molecular Qubits. J. Am. Chem. Soc. 2017, 139, 4338-4341.

(55) Atzori, M.; Benci, S.; Morra, E.; Tesi, L.; Chiesa, M.; Torre, R.; Sorace, L.; Sessoli, R. Structural Effects on the Spin Dynamics of Potential Molecular Qubits. Inorg. Chem. 2018, 57, 731-740.

(56) Yamabayashi, T.; Atzori, M.; Tesi, L.; Cosquer, G.; Santanni, F.; Boulon, M.-E.; Morra, E.; Benci, S.; Torre, R.; Chiesa, M.; Sorace, L.; Sessoli, R.; Yamashita, M. Electronic Spin Qubits into a ThreeDimensional Metal-Organic Framework. J. Am. Chem. Soc. 2018, 140, 12090-12101.

(57) Atzori, M.; Sessoli, R. The Second Quantum Revolution: Role and Challenges of Molecular Chemistry. J. Am. Chem. Soc. 2019, 141, 11339.

(58) Urtizberea, A.; Natividad, E.; Alonso, P. J.; Pérez-Martínez, L.; Andrés, M. A.; Gascón, I.; Gimeno, I.; Luis, F.; Roubeau, O. Vanadyl spin qubit $2 \mathrm{D}$ arrays and their integration on superconducting resonators. Mater. Horiz. 2020, 7, 885-897.

(59) Kandrashkin, Y. E.; Salikhov, K. M.; Stehlik, D. Spin Dynamics and EPR Spectra of Consecutive Spin-Correlated Radical Pairs. Model Calculations. Appl. Magn. Reson. 1997, 12, 141-166.

(60) Salikhov, K. M.; van der Est, A. J.; Stehlik, D. The Transient EPR Spectra and Spin Dynamics of Coupled Three-Spin Systems in Photosynthetic Reaction Centres. Appl. Magn. Reson. 1999, 16, 101134.

(61) Salikhov, K. M.; Zech, S. G.; Stehlik, D. Light induced radical pair intermediates in photosynthetic reaction centres in contact with an observer spin label: spin dynamics and effects on transient EPR spectra. Mol. Phys. 2002, 100, 1311-1321.

(62) Kandrashkin, Y. E.; van der Est, A. Time-resolved EPR spectroscopy of photosynthetic reaction centers: from theory to experiment. Appl. Magn. Reson. 2007, 31, 105-122.

(63) Colvin, M. T.; Carmieli, R.; Miura, T.; Richert, S.; Gardner, D. M.; Smeigh, A. L.; Dyar, S. M.; Conron, S. M.; Ratner, M. A.; Wasielewski, M. R. Electron Spin Polarization Transfer from Photogenerated Spin-Correlated Radical Pairs to a Stable Radical Observer Spin. J. Phys. Chem. A 2013, 117, 5314-5325.

(64) Rugg, B. K.; Krzyaniak, M. D.; Phelan, B. T.; Ratner, M. A.; Young, R. M.; Wasielewski, M. R. Photodriven quantum teleportation of an electron spin state in a covalent donor-acceptor-radical system. Nat. Chem. 2019, 11, 981-986.

(65) Zwanenburg, G.; Hore, P. EPR of spin-correlated radical pairs. Analytical treatment of selective excitation including zero-quantum coherence. Chem. Phys. Lett. 1993, 203, 65-74.

(66) Hol, W. G. The role of the a-helix dipole in protein function and structure. Prog. Biophys. Mol. Biol. 1985, 45, 149-195.

(67) Wallace, P. M.; Sluss, D. R. B.; Dalton, L. R.; Robinson, B. H.; Reid, P. J. Single-Molecule Microscopy Studies of Electric-Field Poling in Chromophore-Polymer Composite Materials. J. Phys. Chem. B 2006, $110,75-82$.

(68) Varela, S.; Montañes, B.; López, F.; Berche, B.; Guillot, B.; Mujica, V.; Medina, E. Intrinsic Rashba coupling due to hydrogen bonding in DNA. J. Chem. Phys. 2019, 151, 125102.

(69) We have assumed here a completely polarized initial state of the qubit, as could be obtained at Q-band at low temperature, but partial population of the excited qubit state only yields an overall reduction of the qubit signal.

(70) Detection of NMR spectrum must be sufficiently faster compared to relaxation/recombination times. Careful choice of the nucleus used as a probe and/or deuteration could be necessary to reduce broadening of the probe peaks due to the interaction with other spins (which must be smaller than $\mathcal{A}$ ) and to isolate the probe Larmor frequency.

(71) Pedersen, J. B.; Freed, J. H. Theory of chemically induced dynamic electron polarization. I. J. Chem. Phys. 1973, 58, 2746-2762.

(72) Edén, M. Computer simulations in solid-state NMR. I. Spin dynamics theory. Concepts Magn. Reson., Part A 2003, 17A, 117-154.

(73) Luo, J.; Hore, P. J. Chiral-induced spin selectivity in the formation and recombination of radical pairs: cryptochrome magnetoreception and EPR detection. New J. Phys. 2021, 23, 043032.

(74) Levanon, H. In Biophysical Techniques in Photosynthesis; Amesz, J., Hoff, A. J., Eds.; Springer Netherlands: Dordrecht, 1996; pp 211233.

(75) Weber, S. Transient EPR. eMagRes 2017, 6, 255-270.

(76) Li, X.; Markandeya, N.; Jonusauskas, G.; McClenaghan, N. D.; Maurizot, V.; Denisov, S. A.; Huc, I. Photoinduced Electron Transfer and Hole Migration in Nanosized Helical Aromatic Oligoamide Foldamers. J. Am. Chem. Soc. 2016, 138, 13568-13578.

(77) Mayländer, M.; Chen, S.; Lorenzo, E. R.; Wasielewski, M. R.; Richert, S. Exploring Photogenerated Molecular Quartet States as Spin Qubits and Qudits. J. Am. Chem. Soc. 2021, 143, 7050-7058.

(78) Bayliss, S. L.; Laorenza, D. W.; Mintun, P. J.; Kovos, B. D.; Freedman, D. E.; Awschalom, D. D. Optically addressable molecular spins for quantum information processing. Science 2020, 370, 13091312. 